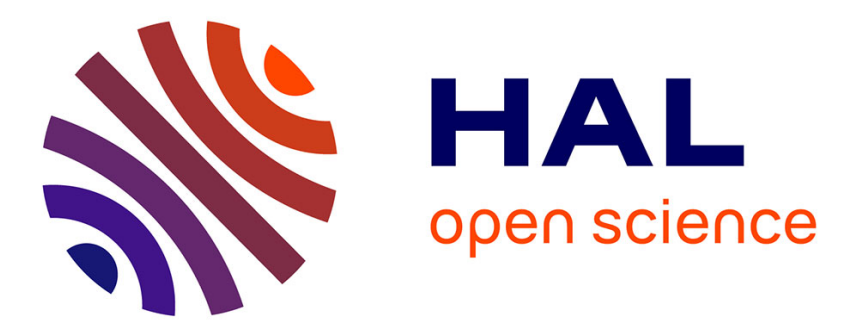

\title{
ENTROPiA: Towards Infinite Surface Haptic Displays in Virtual Reality Using Encountered-Type Rotating Props
}

Victor Mercado, Maud Marchal, Anatole Lécuyer

\section{To cite this version:}

Victor Mercado, Maud Marchal, Anatole Lécuyer. ENTROPiA: Towards Infinite Surface Haptic Displays in Virtual Reality Using Encountered-Type Rotating Props. IEEE Transactions on Visualization and Computer Graphics, 2021, 27 (3), pp.2237-2243. 10.1109/TVCG.2019.2963190 . hal-03139215

\section{HAL Id: hal-03139215 \\ https://hal.inria.fr/hal-03139215}

Submitted on 11 Feb 2021

HAL is a multi-disciplinary open access archive for the deposit and dissemination of scientific research documents, whether they are published or not. The documents may come from teaching and research institutions in France or abroad, or from public or private research centers.
L'archive ouverte pluridisciplinaire HAL, est destinée au dépôt et à la diffusion de documents scientifiques de niveau recherche, publiés ou non, émanant des établissements d'enseignement et de recherche français ou étrangers, des laboratoires publics ou privés. 


\title{
ENTROPiA: Towards Infinite Surface Haptic Displays in Virtual Reality Using Encountered-Type Rotating Props
}

\author{
Víctor Mercado, Maud Marchal and Anatole Lécuyer
}

\begin{abstract}
In this paper, we propose a novel approach towards an infinite surface haptic display. Our approach, named ENcountered-Type ROtating Prop Approach (ENTROPiA) is based on a cylindrical spinning prop attached to a robot's end-effector serving as an encountered-type haptic display (ETHD). This type of haptic display permits the users to have an unconstrained, free-hand contact with a surface being provided by a robotic device for the users' to encounter a surface to be touched. In our approach, the sensation of touching a virtual surface is given by an interaction technique that couples with the sliding movement of the prop under the users' finger by tracking their hand location and establishing a path to be explored. This approach enables large motion for a larger surface rendering, permits to render multi-textured haptic feedback, and leverages the ETHD approach introducing large motion and sliding/friction sensations. As a part of our contribution, a proof of concept was designed for illustrating our approach. A user study was conducted to assess the perception of our approach showing a significant performance for rendering the sensation of touching a large flat surface. Our novel approach could be used to render large haptic surfaces in applications such as rapid prototyping for automobile design.
\end{abstract}

Index Terms - Infinite Surface Haptic Display, Encountered Type Haptic Display, Virtual Environments, Virtual Reality

\section{INTRODUCTION}

$\mathrm{H}$ APTICS represent an important cue in virtual reality (VR) immersion. Haptic sensations are complex and varied, therefore reproducing these represents a challenge. In the sub-field of active haptics, efforts are mostly focused on providing sensations such as contact or collisions with virtual objects [1]. Touching and following a virtual surface naturally (without any constraints) is hard to achieve with traditional approaches, especially with large exploratory motions [2], [3]. Enabling VR users to touch and explore virtual surfaces is still a challenge. Therefore, we propose a novel approach that enables to touch and explore virtual surfaces with unconstrained and large exploratory procedures that permit to exploit an alternative approach for encountered-type haptic displays (ETHDs). This type of displays consists on robotic actuators that permit to display a surface by encountering the user's hand position [4]. This configuration permits a free-hand interaction in multiple spatial points. ETHDs aim to provide an unconstrained experience at the moment of touching rendered surfaces in a virtual environment (VE).

However, state-of-the-art in ETHDs approaches for haptic rendering have a constrained contact area primarily limited to the size of the display being held by the device. This issue reduces the exploration area at the moment of interacting in a VE where a ETHD is integrated. While the ETHD research community has been exploring alternatives for rendering haptic feedback in a wider variety of spatial

- V. Mercado works at Univ Rennes, INSA, Inria, CNRS, IRISA

- M. Marchal works with Univ Rennes, INSA, Inria, CNRS, IRISA

- A. Lecuyer works with Univ Rennes, Inria, CNRS, IRISA E-mail: \{victor.mercado,maud.marchal, anatole.lecuyer@inria.fr\} points through mobile devices, the challenge of increasing the contact area for ETHDs remains open [2]. Additionally, the necessity of having multiple textures to be provided as a part of haptic feedback is gaining importance for increasing the diversity of rendered surfaces [3], [5], [6].

In this paper, we propose a novel ENcountered-Type ROtating Prop Approach (ENTROPiA) for displaying haptic virtual surfaces based on ETHD without the constraint of a limited surface area to be in contact with. Another term proposed in this work is infinite surface haptic display (ISHD). Our approach relies on a rotating surface as a mean to display a large surface (see Figure 1). This approach relies on having the users to be in contact with a spinning round surface that a robotic system can position and rotate according to the users' hand location. The sensation of touching a flat surface is given by the sliding movement of the prop under the finger and the usage of an interaction technique that exploits this approach. The interaction takes place in a VE using a head mounted display (HMD) and a finger tracking system. Users are able to touch different textured surfaces in a VE by following a trajectory that permits them to explore the surface with their sense of touch.

The contributions of this paper are:

1) A novel approach for unconstrained large surface haptic rendering leveraging ETHDs and introducing a rotating prop.

2) A proof of concept that illustrates our proposed approach.

3) A user study assessing haptic perception provided by our approach and comparing it to state-of-the-art on large shape rendering techniques for ETHDs. 


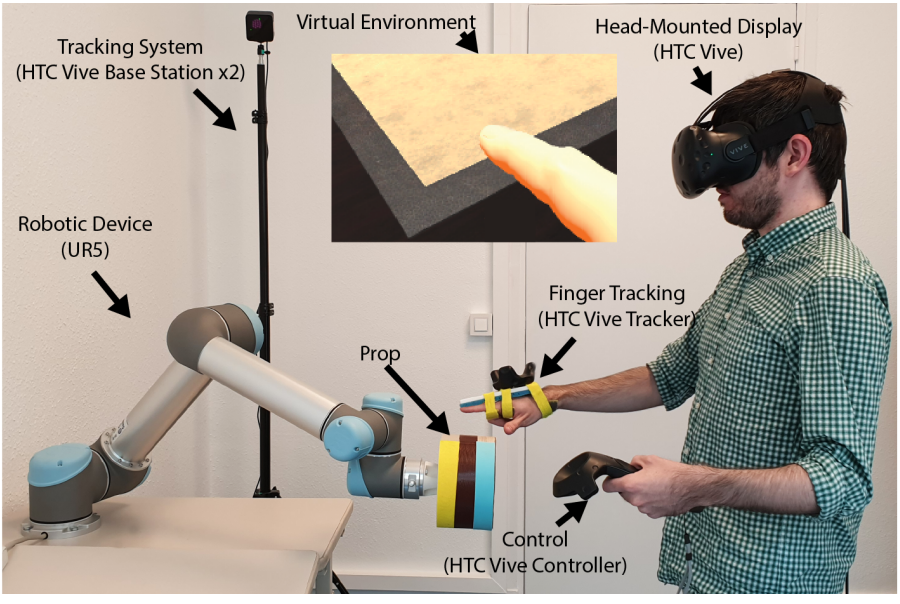

Fig. 1. Global setup of our system. Our proposed rotating prop (bottomcenter) can provide a large haptic surface display together with different textures represented in a VE (top-center).

The remaining of this paper is organized as follows: Related work is discussed in Section 2. Our approach is introduced in Section 3 along with its main components. Section 4 presents the design of a proof of concept that contextualizes the contribution of this paper. Later, Section 5 describes a user study conducted for evaluating the rendering perception for our approach. Results of our approach performance in the user study are discussed in Section 6 . The paper ends with a general discussion.

\section{Related Work}

This section covers related work concerning primarily ETHDs and the different types of alternatives being considered by researchers to enhance these devices in matters of texture and surface rendering. The concept of ETHDs and their premise is introduced in the first part of this section. Later, related work on grounded and unconstrained ETHDs concerning shape and texture rendering is discussed. To finalize this section, related work trying to pursue an approach similar to infinite surface rendering is presented.

\subsection{Encountered Type Haptic Displays}

ETHDs are based on the premise of providing a free-hand contact through the coupling of a tracking system and a actuator [5]. The former constantly tracks the user's position and provides the information to the actuator to position a surface to be touched in a manner that the user can encounter and touch a surface displayed in a VE. For achieving this sensation, ETHDs have been relying on robotic systems, tangibles and actuators as a mean to provide haptic feedback to the users only when they voluntarily decide to touch or interact with an element in a virtual simulation. Early implementations of these systems consisted on robotic actuators holding a tangible interface that a user could touch in a VE [4], [7], [8], [9].

The ETHD field evolved through the first decade of 2000s seeking new options for rendering different shapes and volumes. The democratization of HMDs technologies in the early 2010s led to further exploration in rendering techniques and applications for ETHDs. For instance, Filippeschi et. al. developed a juggling simulation by using a ETHD system with 2 haptic devices and a 3D projection screen [10]. The application of these systems have also been considered for virtual therapies such as the fear of heights in the work of Brice et. al. [11].

\subsection{Grounded Haptic Displays}

Grounded ETHDs based on robotic arms have been exploring the possibilities of enhancing their end-effectors as a mean to provide enriched haptic feedback. The work of Araujo et al. [6] contributed in the ETHD field by having a multi-sided end-effector as way to increase the type of surfaces displayed in a VE in matters of texture, buttons and functionality. Posselt et. al. [5] used a multi-sided endeffector for exploring the possibilities of rendering different textures in a VE which simulates a car cockpit. In the designed VE, users are able to touch materials such as leather, fabric, and plastic. Yamaguchi et. al. [3] developed a system based on several textured panels in order to provide a greater contact area. The system implemented by the research team had the purpose of recreating a virtual house where parts of it with different materials could be touched.

The ETHD field has nurtured from both active and passive haptic approaches along with progress on robotics, display technologies and control algorithms [12]. Despite that fact, most of the work presented still has the issue of having a limited contact area regardless of the system positioning capability.

\subsection{Unconstrained Haptic Displays}

In recent years the usage of robotic systems has expanded considering ungrounded systems which has been classified as unconstrained haptic displays (UHDs). In this category we can consider wheeled-pin arrays and unmanned autonomous vehicles (UAVs) such as drones.

UAVs have the advantage to move freely to many locations surrounding the user. Research work in [13], [14], [15], [16], [17], [18] has profited from the integration of drones in to VEs to become tangible objects which encountered the user according to the task being realized. Researchers have also used the characteristics of drones such as the airflow generated by the rotors to produce a sensation of stiffness to the surface being held by the vehicle [13], [15]. Wheeledpin displays have the advantage of possessing an active shape rendering mechanism and the capability of displacing themselves in two dimensions [19], [20]. Currently, these systems are still constrained to the actuators holding the pin-array.

Present work in UHDs has not considered to increase the contact area. In terms of UAVs, constraints of rotation and stiffness are still opportunity areas on the field. We observe that wheeled pin-arrays are also aiming for providing an optimal haptic display [19]. Nevertheless, the constraint of resolution and systems' positioning are still being addressed by researchers.

\subsection{Towards Infinite Surface Haptic Displays}

Recent efforts made by the ETHD community have been addressing the issue of having a limited contact area. The 
presented research aims to increase the surface that can be touched once the encountered haptic display is integrated in a virtual simulation.

The work form Whitmire et. al. [2] addresses the issue of having a limited contact area and developed a haptic revolver. This device presents the concept of having a hand grip integrating a cylindrical spinning surface. The hand grip possesses an actuator system which tilts the cylindrical surface to get in contact with the users' finger exclusively when it is touching an element in the simulation. The surface then starts spinning as users displace their finger for touching elements in a VE. For instance, the authors designed a card game scenario where users could touch the fabric covering a poker table or the cards material. This work eliminates the limited surface constraint leading research into ISHDs. While this research work presumably introduced a new paradigm in haptics, a familiar constraint is present: the need of always holding or touching the rendering device.

Vonach et. al. [21] also used a passive textured surfaces for recreating a virtual wall in a virtual simulation. In their work, they profit from the usage of a omni-directional treadmill for providing haptic feedback in several locations of the VE. In this work, the constraints of the grounded ETHD were overcame by designing an infinite VE which was optimized to have elements that could be touched according to the position and direction of the user in the treadmill. While the contact with the surface is not continuous, this work demonstrates that with an optimal setup, a grounded ETHD can be as useful as a UHD.

\section{OUR NOVEL APPROACH FOR AN ETHD-BASED INFINITE SURFACE HAPTIC DISPLAY : ROTATING PROPS}

Our objective is to provide a large haptic surface rendering that could benefit from the ETHD property of having an unconstrained, free-hand interaction. However, current limitations on the ETHD field are mainly based on not having a large surface that could be touched in a continuous, natural manner. To do so, we leverage a grounded ETHD by providing a larger contact area rendered by the integration of a rotating prop and an interaction technique that permits to touch a defined, virtual surface.

The principle (see Figure 2) relies on having the user to be in contact with a spinning round surface which a robotic system can position and rotate according to the users' hand location. The sensation of touching a flat surface is given by the sliding movement of the prop under the finger and the usage of an interaction technique that exploits this approach. As the finger displaces while touching the surface, the round prop rotates and displaces in the same trajectory to always provide an area to be in contact with. Profiting from its curvature, the round prop rotates on the opposite direction of its displacement thus creating the sensation of continuously touching a large flat surface without the necessity of physically having a surface of the same shape and dimensions as currently done in stateof-the-art approaches. Additionally, this principle permits to increase the contact area of a traditional encountered haptic device by compensating their actual mobility range and end-effector size and volume as a mean to render any required surface.

The prop can be either integrated to a robotic arm (see Figure 3) but can also be attached directly to the haptic device or can be integrated with the aid of a spinning motor.
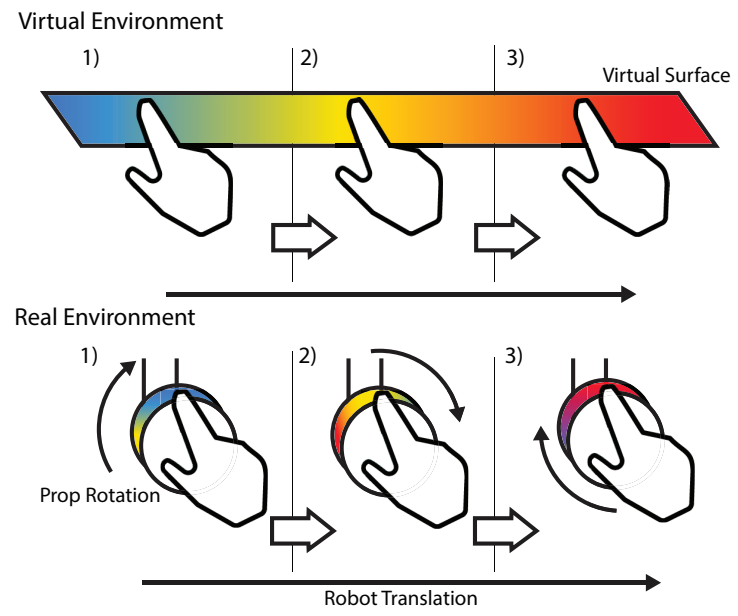

Fig. 2. ENTROPIA's principle. The sensation of touching a flat surface in a VE is given by touching the rotating prop and simultaneously displacing it through the same trajectory and speed of the users' finger. Considering the prop's size and shape as well as both translation and angular speed, users are always in contact with a "flat face" that continuously renders itself at the same time when they displace their finger.

The key components of our approach are:

1) Encountered-type haptic display composed of a grounded Universal Robots UR5 robotic arm, a Vive HMD for displaying the virtual environment, a Vive tracker and base stations for tracking users' hand position and a Vive controller for controlling the simulation (See Figure 1).

2) A cylindrical prop containing textures all over its circumference, giving the sensation of touching an infinite surface while rotating below users' fingers (See Figure 3).

3) An interaction technique to guide users when performing the exploratory haptic procedure.

The integration of these components enables:

- Large surface rendering: our system enables the sensation of exploring a large flat surface by spinning and positioning our cylindrical prop under the users' finger location.

- Multiple texture rendering: the proposed prop can integrate multiple textures which can be applied to infinite surface rendering mode. The textures are integrated into our approach by several textured stripes that surround the curved side of the prop. When covering the entire circumference of the curved part, we can render an infinite textured surface as long as the system can locate the prop and spin it under the user's finger.

\subsection{Rotating Props}

Our prop permits to have contact over its curved side. By spinning the cylindrical prop the robot keeps the user in 


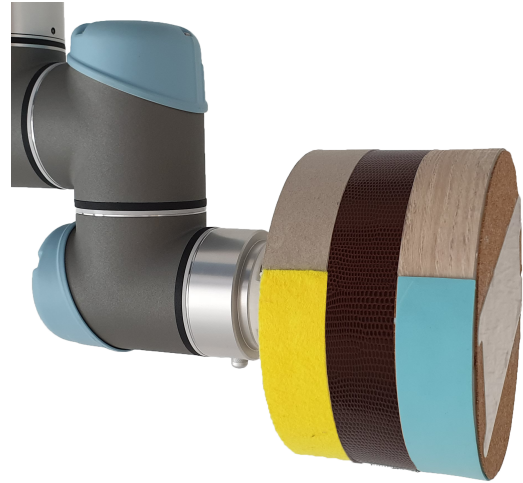

Fig. 3. The designed cylindrical prop for representing our approach. This prop can hold different textures which are used to render flat surface in a VE. Textures as cork, foam, felt, leather, cardboard and wood are displayed on this figure. In this case, the leather texture covers the prop's whole circumference, this permits to render this texture in an infinite way as long as the prop spins below users' finger.

contact with a face of the prop. As long as the user follows the contact area, the surfaces to be touched are only limited by the range of mobility of the robotic device rather than the overall volume and area of the prop itself.

The prop's height permits to include several textures over its surface which can cover the whole circumference or just a part of it for having a shorter contact area but for increasing the texture range. The influence of the prop's radius permits to render the sensation of touching a flat surface while spinning since touching with one finger a part of its circumference does not disclose the prop's cylindrical shape. Therefore, it is possible to obtain a contact area greater that the prop's diameter by rotating and displacing it under the users' finger.

\subsection{Interaction Technique}

Our exploratory interaction technique relies primarily on having a contact area that moves on a predefined trajectory. The contact area is conceived for a one-finger interaction to couple with the geometrical properties of our prop for rendering the sensation of touching a flat surface. Our interaction technique is inspired by the work presented by Posselt et. al. [5] in which the authors propose a technique for profiting from the workspace given by a polyhedral ETHD prop. This technique consists on displaying the possible areas that user could touch in a multi-textured VE simulating a car cockpit for automobile design. Once the user chooses an area the grounded ETHD system placed a face of the prop to that area that corresponded with the texture of the virtual object. Users were always informed of the available touch workspace with the aid of a visual cue that signaled the borders and shape of the polyhedral prop's face. Currently, state-of-the-art in ETHDs proposes interaction techniques that limit the contact to the area of their device's end-effector. In our interaction technique, a colored surface is displayed on a VE where the user can touch the contact area represented by a square. The squared contact area was designed to guide the users and to avoid that they slide to an undesired part of the prop and thus mismatching what they see in the VE and what they feel with their sense of touch. Users are asked to touch the squared area with their index finger as a mean to optimize hand and finger tracking and to have a contact area proportional to the size of the prop (see Figure 4).

Our exploratory interaction technique has three phases:

1) Ready Phase. The contact area is colored orange and users are prompted to move their finger from the resting location to touch the contact area.

2) Touch Phase. The contact area is colored blue and participants are asked to touch and follow this surface. The contact area is displaced over a virtual surface matching the trajectory of the passive prop surface.

3) Release Phase. The contact area turns red and users are prompted to release the finger from the surface and maintain it around the waist level for avoiding unexpected contact with the prop if re-positioning the robot's end-effector.

Thanks to our interaction technique, the user is permitted to perform an haptic exploratory procedure in a defined area of a VE. This technique integrates with our proposed prop for increasing the contact area of a haptic area and thus moving towards the ISHD.

\section{Proof of Concept}

We designed a proof of concept to showcase the possibilities of ENTROPiA. This section comprises the description of the hardware we used to implement this proof concept as well as the scenario that contextualizes our approach and the virtual environment that integrates both the system implementation and the scenario metaphor.

\subsection{Setup}

The system was implemented with a Universal Robots UR5 robotic arm. This robot was programmed with several movement routines for displacing and/or rotating the endeffector for recreating a flat surface. Users have contact with the surface with the tip of their right index finger. Participants used their left hand for using the Vive controller for navigating through the experiment user interface.

The system uses a HTC Vive HMD tracker and controller for displaying an controlling the virtual simulation. The Vive tracker was attached to a support which constrained finger movement on the right hand for only letting the user to touch the prop with the index finger (see Figure 8). Robot's communication with Unity was achieved through TCP port communication and parsing commands to the UR5 programming language and interface. The VE was implemented on a computer with an Intel Core i7-7820HQ processor with an NVIDIA Quadro P3000 graphics processor.

The prop designed consists in a cylindrical shape with a radius of $8.4 \mathrm{~cm}$ and $10 \mathrm{~cm}$ in height. The prop was displaced $17 \mathrm{~cm}$ to the right at a speed of 0.05 m.s. When rotating, the prop had an angular velocity of $0.2 \pi$ rad.s. 


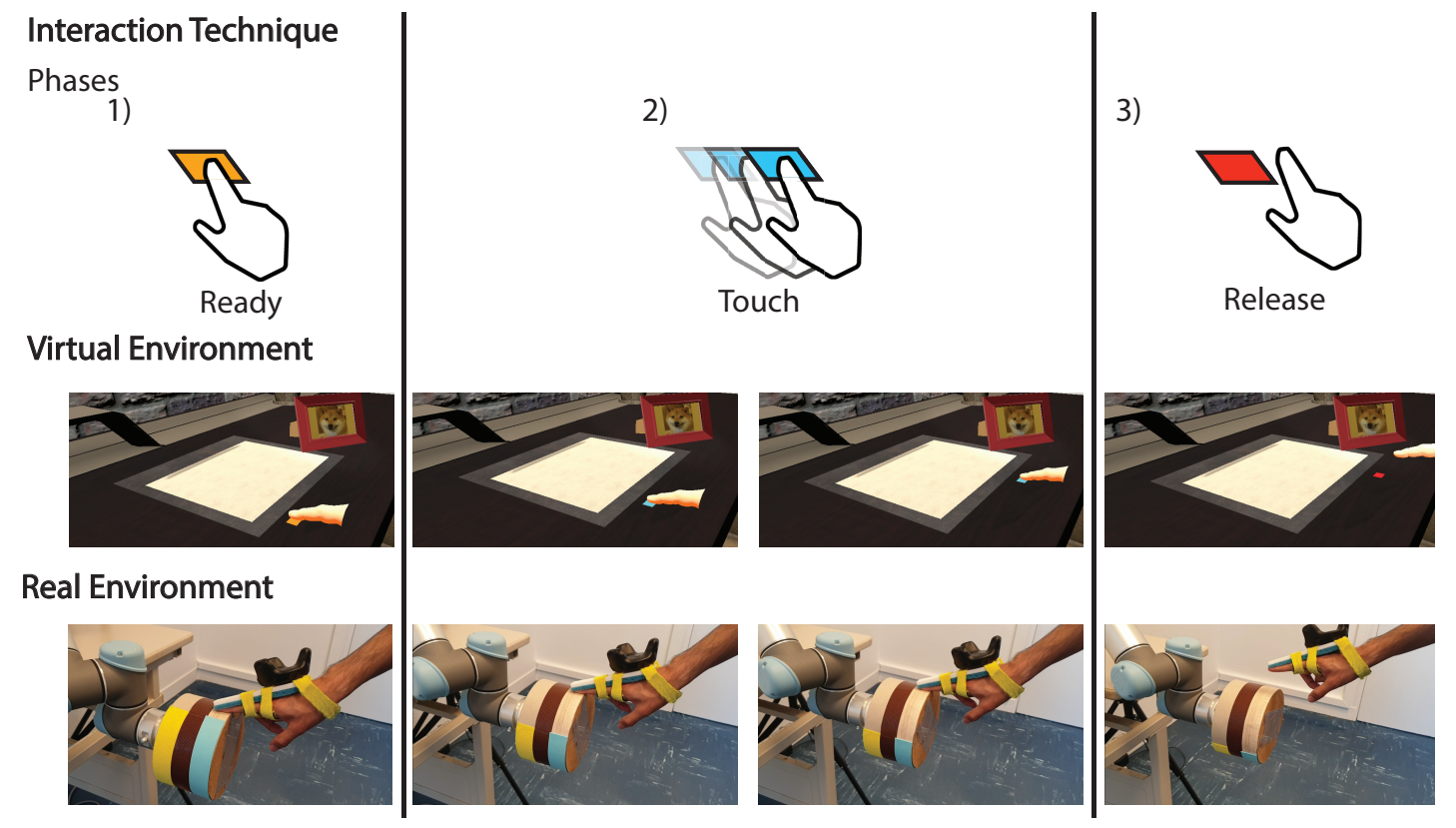

Fig. 4. Interaction technique. This technique enables the user to explore a surface and its texture in a VE. The phases of the technique are represented both in the VE and in real life with the prop. The contact area is represented by a colored square that displaces over a surface in our VE. On the ready phase the contact area becomes orange telling the user to be ready for movement and to touch the square. On the touch phase, the colored square becomes blue and displaces over the surface. The user is in constant contact with the surface in this phase as a mean to haptically explore the surface. The release phase prompts users to lift their finger to stop the contact with the device.

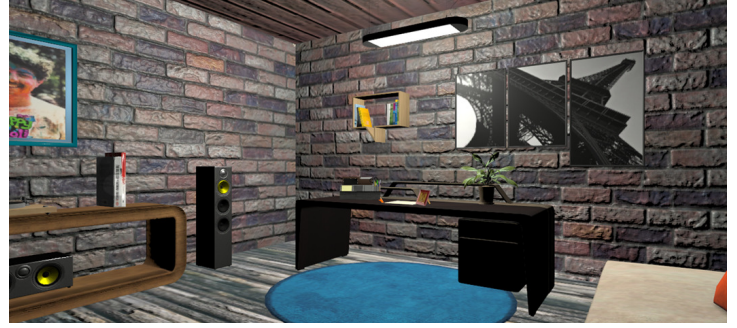

Fig. 5. The scenario setup for the studio. Our approach is integrated in this virtual scenario to render the sensation of the textures located on the desktop.

\subsection{Scenario}

The VE displays a studio where different flat textures can be explored (see Figure 5). A classic covered desktop was chosen as the main feature to be touched in this scenario since it portraits different textures that are frequently found in that context. In this environment, the user can touch four different materials: the desktop wood, a leather cover for the desktop, a sheet of paper for representing documents and planes and the glass of a photo frame. The materials were assigned to the different elements present in the environment. The wood was assigned to the desktop while leather, paper and tempered glass were assigned to a desktop cover, a print and a photo frame respectively.

\section{USER StUdy}

We conducted a user study in order to assess haptic perception of virtual surfaces simulated by our approach and compare with state-of-the-art surface rendering techniques as well as the ground truth perception.

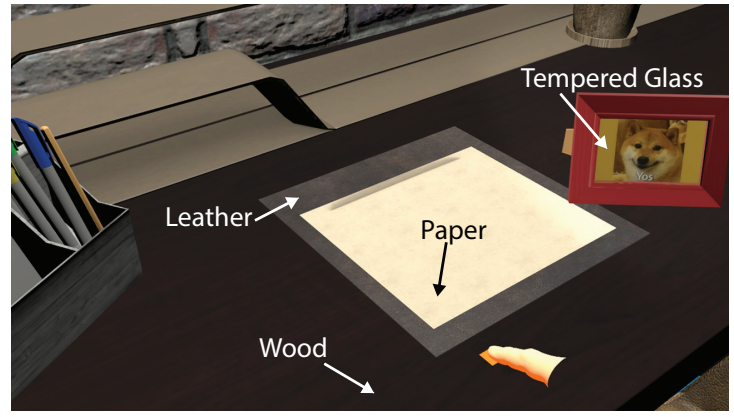

Fig. 6. Details of the virtual scenario. The VE was designed to mimic a desktop in a studio where different textures could be touched in different objects such as a wooden desktop, a leather cover, and a sheet paper. The photo frame could also be touched by using the state-of-the-art approach.

\subsection{Experimental Design}

Four surface haptic display methods were considered in our experimental design. These display methods consider both ours and state-of-the-art approaches to be compared against the ground truth as a way to determine which approach renders haptic feedback that could be perceived as touching a flat surface. The four haptic display methods were the following:

- Ground truth (GT): A large flat surface is touched and used as a reference for comparing to the other type of surfaces rendered. The GT is represented as the largest part of the experimental prop.

- Translating Flat Surface (TFS): The smallest rectangular side of the experimental prop was used as the contact area. The surface was displaced but not rotated. This haptic display represents the current 
state-of-the-art approach since it primarily considers the flat end-effectors commonly used in ETHD reported literature.

- Translating and Rotating Round Surface (TRRS): This condition represents ENTROPiA. One side of the cylindrical part of the prop was used as the contact area. The surface was displaced and rotated. This haptic display represents our approach of increasing a haptic device contact area without the necessity of having a large physical surface.

- Translating Round Surface (TRS): One side of the cylindrical part of the prop was used as the contact area. The surface was displaced but not rotated. This haptic display represents an intermediate phase between the state-of-the-art and our proposed approach. The user touched one part of the experimental prop's circumference while the latter was displaced by the robotic system. This haptic display was considered to evaluate if users could perceive a flat surface by touching curved non-rotating surface.

The hypothesis of our experiment was the following: the sensation of touching a flat surface can be obtained if users displace their finger over the round part of a cylindrical shape while the latter is spinning and moving.

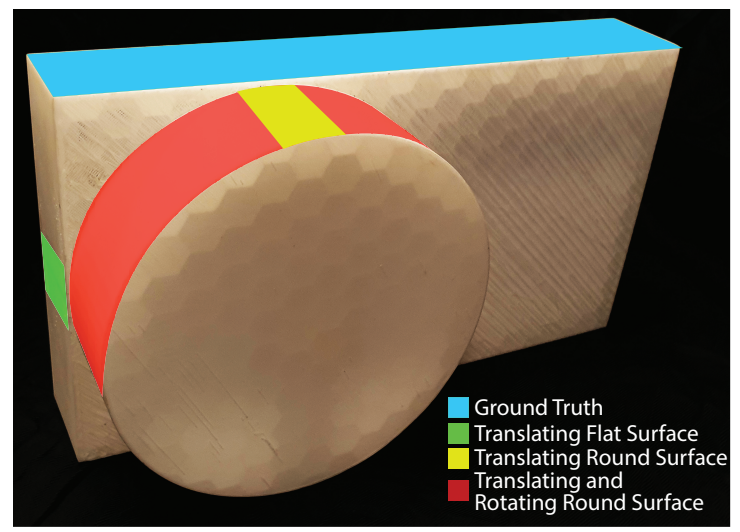

Fig. 7. The experimental prop. A rectangular prism was attached to the back of our proposed prop as a mean to have different alternatives to be compared against the GT which is depicted on the longest side of the prop. For the TFS users touched the shortest flat surface of the prop. The TRS is represented by one small part of the prop's circumference. The TRRS represents our novel approach and comprises the whole cylindrical surface that users touched while the prop translated and rotated at the same time. This shape recreates the same approach used in our proof-of-concept.

\subsection{Experimental Setup}

A multi-sided prop was designed in order to comprise on different conditions, compared against the sensation of touching a flat surface representing the ground truth. The experimental prop possesses a cylindrical shape that measures $8.4 \mathrm{~cm}$ in radius and $10 \mathrm{~cm}$ in height attached to a rectangular measuring $30 * 15.5 * 5 \mathrm{~cm}$. The longest face (see Figure 8 bottom-right) is used to represent the ground truth. The shorter face of the prism was used to depict the ETHD's state-of-the-art static surface approach. The cylindrical part of the prop is also attached to represent the ENTROPiA approach.

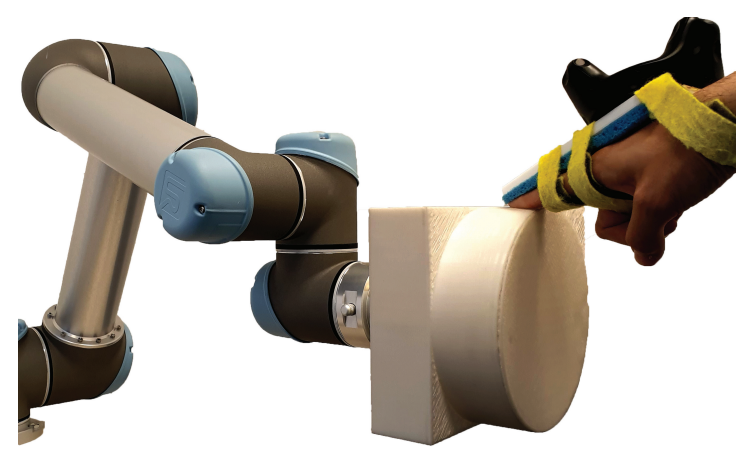

Fig. 8. Experimental setup. The hand support used for fixing the users' hand position and for sustaining the Vive tracker (upper right) and the multi-sided experimental prop with a flat surface integrated (center) this experimental prop was displaced and rotated by a robot unit (left) for having an ETHD-based approach.

\subsection{Procedure}

Before getting involved in the experiments, participants were asked to fill a consent form and were informed that they were going to interact with a robotic device. Participants were equipped with a headset for listening to white noise when doing the experiment in order to not perceive sounds produced by the robot while displacing the endeffector.

Each trial of the experiment comprised two stimuli. The first stimulus or the ground truth consisted on touching the longest flat side of the experimental prop. Then, a second stimulus consisted on one of the other three haptic displays of the experiment: TFS, TRS and TRRS. At the end of each trial, users were asked to qualify the perception similarity between the stimuli presented in a Likert-scale from 1 (completely different) to 7 (completely similar). These display methods were presented in a random order 10 times per approach, making a total of 30 trials.

\subsection{Collected Data and Population}

We collected data concerning the perceived similarity between the two stimuli presented on each trial. After completing the experiment, participants were asked to fill a questionnaire which assessed their subjective opinion about any possible factor that could have influenced their perception during the trials. The questions concerned the factors that could have helped the users to differentiate between the ground truth and the alternative haptic rendering techniques: did motion $(\mathbf{Q 1})$, curvature $(\mathbf{Q} 2)$ and speed $(\mathbf{Q} 3)$ helped you to differentiate the two stimuli? The questions were presented in a Likert-scale answer format ranging from 1 (completely disagree) to 7 (completely agree). In the end, participants were asked to name another factor (if existed) which could have helped them to compare both stimuli.

Twelve participants ( 3 females and 9 males, $M=24.58$ ) took part in the experiment. Two of them were left-handed.

\subsection{Results}

\subsubsection{Experiment}

To study the perception similarity between the different stimuli, we first analyzed the ratings for the answers concerning the comparisons between the GT and the three 
other haptic displays. We used a linear mixed model on the collected data to model the probability of perceived similarity with respect to the haptic display variable. The participants are considered as a random effect in the model. We performed an analysis of variance of the model and we found a significant effect of the display $(F(2,346)=79.11$, $p<0.001$ ). We performed a post-hoc analysis on the haptic displays using a Tukey test. Bonferroni correction was used for all post-hoc analysis. We found a significant difference between all the conditions: ENTROPiA (TRSS, $M=4.39$, $S D=1.72)$ obtained higher ratings than TFS $(M=2.79$, $S D=1.55, Z=9.01, p<0.001)$, and than RS $(M=2.24$, $S D=1.47, Z=12.11, p<0.001)$. TFS was significantly rated higher than $\operatorname{RS}(Z=3.10, p=0.006)$.

Participants responses are displayed in Figure 9.

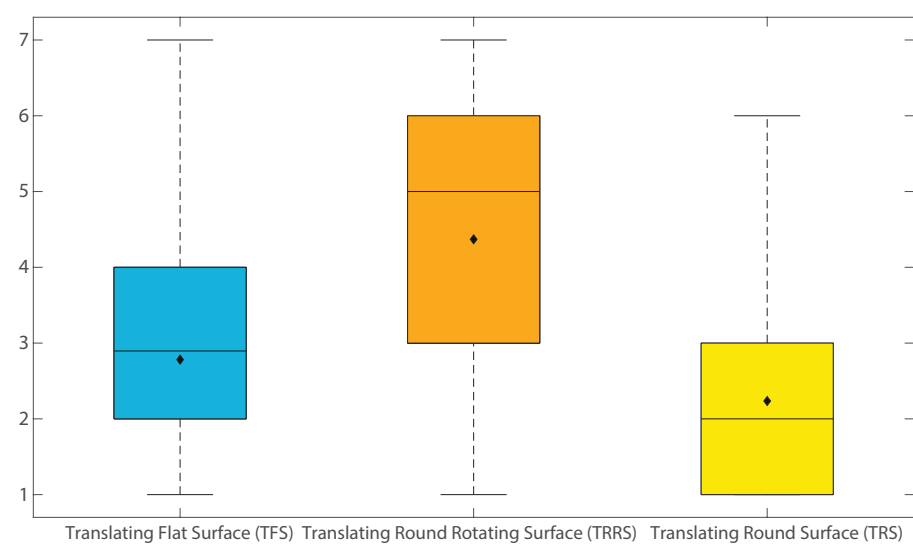

Fig. 9. Participant's responses for assessing the similarity between touching the ground truth and the other three continuous contact rendering techniques. The round rotating surface was evaluated as the closest medium for providing a sensation closer to the ground truth.

\subsubsection{Questionnaire}

According to responses to Q1, participants considered that the sensation of motion was important for differentiating the two stimuli $(\mathrm{M}=5.666, \mathrm{SD}=1.874)$. Results from $\mathrm{Q} 2$ suggest that curvature was useful to differentiate the stimuli $(\mathrm{M}=4.75, \mathrm{SD}=1.712)$. The answers from $\mathrm{Q} 3$ demonstrated that the perceived speed was determinant for identifying differences between the ground truth and the alternative continuous surface rendering techniques $(\mathrm{M}=5.833, \mathrm{SD}=$ 1.466).

\section{Discussion}

The design of ENTROPiA was conceived for addressing the necessity of having a free-hand haptic exploratory procedure that could not be limited to the display's size and shape properties. In order to achieve this objective, we designed a rotating cylindrical prop that functions as an ETHD through an interaction technique that permits to explore a virtual surface coping the prop's rotation and position to provide the sense of touching a flat surface under the users' finger. A proof of concept and a user study were carried to test the integration of our approach in to a VE an to test its effectiveness at the moment of rendering haptic feedback.

The proof of concept integrates different textures for flat surface rendering in a virtual simulation. The premise of the multi-sided end-effector contributed by [5], [6] is retrieved in our approach by having an assort of textures placed over the cylindrical prop's curved surface. Integrating a texture that could cover the entire curved surface's circumference could lead to an infinite surface rendering due to the surface's geometrical properties.

The user study was elaborated with the purpose of assessing and comparing users haptic perception between touching a flat surface and several approaches for rendering a similar sensation including our approach, one based from state-of-the-art, and a mix of both. Results were in favor of our rotating prop approach as a mean to render a sensation closer to touching the ground truth. Participants helped for determining important factors that influence shape perception. Speed, curvature and motion were the most influential factors for differentiating the sensation perceived by touching the ground truth and the other three conditions of the experiment. Two out of twelve participants mentioned that they perceived differences in the texture between the ground truth and the other conditions. The rest of the participants did not find any other factor beyond motion, curvature and speed that could have been useful for them to differentiate between the two stimuli.

The contact area was reduced in order to have proper the sensation of touching a flat surface. A bigger prop might imply a larger contact area yet the size might constraint the robot. Exploration in terms of influence of radius could determine if the aforementioned assumption is correct. Further work could also explore rendering curved surfaces and texture friction influence in haptic perception using the our approach. The design of the VE served to assign an application to our approach. Our proposed prop could hold even more textures depending on the interaction design of the environment where this approach could be integrated. With our approach the user is now able to naturally explore a surface rather than having limits according to the faces of a polyhedral prop or the resolution of a pin-array.

The optimal parameters of angular velocity and prop radius might vary from user to user. A personalized speed calibration session might be needed to find an optimal velocity for providing the sensation of touching a flat surface.

Adapting a rotating round prop on a grounded ETHD provides the possibility of rendering a virtual surface while having a natural haptic exploratory procedure. Nevertheless, it could be also considered for other types of ETHDs. For instance, our approach could be incorporated to a mobile device as a mean to have an unconstrained, continuous haptic feed which might become considerably close to the ideal haptic device. Following the premises of the movable surface for rendering different volumes such as the work of Abtahi et. al. [1], our approach does not need an active haptic display to render different volumes. Additionally, our approach could benefit from other contributions to the ETHD field. Including an omni-directional platform approach by Vonach et. al. [21] combined with the system setup proposed in this paper could provide an immersive and realistic haptic experience. Integrating our approach to an UHD such as a drone could also be an alternative to move towards to the infinite surface haptic display. 


\section{CONCLUSION}

ENTROPiA integrates the benefit of having a free-hand interaction provided by an ETHD system and at the same addresses these type of devices pitfall concerning the size of contact area for haptic exploration. A cylindrical prop and a novel interaction technique were conceived as a mean to optimize the size of a multi-textured end-effector as well as the working area of a grounded ETHD-based system. The system functions by displacing and rotating the prop below the user's finger to always provide a surface to be touched, giving the illusion of being in contact with a flat surface when following the prop's trajectory. A proof of concept ETHD was provided to explore several objects with different textures such as the case of a wooden desktop, a leathered cover and a sheet of paper. A user study was conducted to evaluate the haptic perception of virtual surfaces simulated by our approach. Participants responses were in favor of our approach as the mean to render a sensation closer to touching the ground truth.

Future work will comprise further exploration both in surface rendering and interaction techniques for our approach. Radius influence will be further studied with a multi-radii prop for assessing performance in surface rendering. Additionally, our approach rendering capabilities for curved surfaces will be explored by exploiting the prop's geometrical properties. To increase our approach applications, we will design and propose new interaction techniques that could permit to render a larger and wider contact area. Additionally, we will work in the integration of other scenarios for our novel approach.

The results from the user study suggest that we could further explore this approach for surface rendering in ETHDs. The premise of increasing the working area of an haptic display through our approach could be taken into account for fast prototyping in the field of industrial design as shown in [5] yet this approach could also be retrieved by the community as a mean to enhance and optimize their ETHD approaches and moving towards the phase of infinite surface rendering.

\section{ACKNOWLEDGMENTS}

The authors would like to thank the Agence Nationale de la Recherche (ANR) for the grant for funding the LobbyBot project.

\section{REFERENCES}

[1] P. Abtahi and S. Follmer, "Visuo-Haptic Illusions for Improving the Perceived Performance of Shape Displays," in Proc of the CHI Conference on Human Factors in Computing Systems, 2018, pp. 1-13.

[2] E. Whitmire, H. Benko, C. Holz, E. Ofek, and M. Sinclair, "Demonstration of Haptic Revolver: Touch, Shear, Texture, and Shape Rendering on a VR Controller," in Extended Abstracts of the CHI Conference on Human Factors in Computing Systems, 2018, pp. 1-4.

[3] S. Yamaguchi, H. Shionoiri, T. Nakamura, and H. Kajimoto, "An Encounter Type VR System Aimed at Exhibiting Wall Material Samples for Show House," in Proc of the ACM International Conference on Interactive Surfaces and Spaces, 2018, pp. 321-326.

[4] Y. Yokokohji, R. Hollis, and T. Kanade, "What you can see is what you can feel-development of a visual/haptic interface to virtual environment," in Proc of the IEEE Virtual Reality Annual International Symposium, 1996, pp. 46-53.
[5] J. Posselt, L. Dominjon, A. Bouchet, and A. Kemeny, "Toward virtual touch: investigating encounter -type haptics for perceived quality assessment in the automotive industry," in EuroVR Industrial Track, 2017.

[6] B. Araujo, R. Jota, V. Perumal, J. X. Yao, K. Singh, and D. Wigdor "Snake Charmer: Physically Enabling Virtual Objects," in Proc of the 10th International Conference on Tangible, Embedded, and Embodied Interaction, 2016, pp. 218-226.

[7] K. Hirota and M. Hirose, "Development of surface display," in Proc of IEEE Virtual Reality Annual International Symposium, 1993, pp. 256-262.

[8] S. Tachi, T. Maeda, R. Hirata, and H. Hoshino, "A Construction Method of Virtual Haptics Space," in Proc of the 4th International Conference on Artificial Reality and Tele-Existence, 1994, pp. 131-138.

[9] W. McNeely, "Robotic graphics: a new approach to force feedback for virtual reality," in Proc of IEEE Virtual Reality Annual International Symposium, 1993, pp. 336-341.

[10] A. Filippeschi, F. Brizzi, E. Ruffaldi, J. M. Jacinto, and C. A. Avizzano, "Encountered-type haptic interface for virtual interaction with real objects based on implicit surface haptic rendering for remote palpation," in Proc of the IEEE/RSJ International Conference on Intelligent Robots and Systems, y, 2015, pp. 5904-5909.

[11] D. Brice, S. Devine, and K. Rafferty, "A Novel Force Feedback Haptics System with Application in Phobia Treatment," in Proc of the 25th International Conference on Computer Graphics, Visualization and Computer Vision, 2017.

[12] Y. Kim, H. J. Kim, and Y. J. Kim, "Encountered-type haptic display for large VR environment using per-plane reachability maps: Encountered-type Haptic Display for Large VR Environment," Computer Animation and Virtual Worlds, vol. 29, no. 3-4, 2018. [Online]. Available: http://doi.wiley.com/10.1002/cav.1814

[13] K. Yamaguchi, G. Kato, Y. Kuroda, K. Kiyokawa, and H. Takemura, "A Non-grounded and Encountered-type Haptic Display Using a Drone," in Proc of the Symposium on Spatial User Interaction, 2016, pp. 43-46.

[14] P. Knierim, T. Kosch, A. Achberger, and M. Funk, "Flyables: Exploring 3d Interaction Spaces for Levitating Tangibles," in Proc of the International Conference on Tangible, Embedded, and Embodied Interaction, 2018, pp. 329-336.

[15] M. Abdullah, M. Kim, W. Hassan, Y. Kuroda, and S. Jeon, “HapticDrone: An Encountered-Type Kinesthetic Haptic Interface with Controllable Force Feedback: Initial Example for 1d Haptic Feedback," in Adjunct Publication of the 30th Annual ACM Symposium on User Interface Software and Technology, 2017, pp. 115-117.

[16] P. Knierim, T. Kosch, V. Schwind, M. Funk, F. Kiss, S. Schneegass, and N. Henze, "Tactile Drones - Providing Immersive Tactile Feedback in Virtual Reality through Quadcopters," in Proc of the CHI Conference Extended Abstracts on Human Factors in Computing Systems, 2017, pp. 433-436.

[17] M. Hoppe, P. Knierim, T. Kosch, M. Funk, L. Futami, S. Schneegass, N. Henze, A. Schmidt, and T. Machulla, "VRHapticDrones: Providing Haptics in Virtual Reality through Quadcopters," in Proc of the 17th International Conference on Mobile and Ubiquitous Multimedia, Nov. 2018.

[18] P. Abtahi, B. Landry, J. J. Yang, M. Pavone, S. Follmer, and J. A. Landay, "Beyond The Force: Using Quadcopters to Appropriate Objects and the Environment for Haptics in Virtual Reality," in Proc of the CHI Conference on Human Factors in Computing Systems, 2019, pp. 1-13.

[19] D. Fitzgerald and H. Ishii, "Mediate: A Spatial Tangible Interface for Mixed Reality," in Extended Abstracts of the CHI Conference on Human Factors in Computing Systems, 2018, pp. 1-6.

[20] A. F. Siu, E. J. Gonzalez, S. Yuan, J. Ginsberg, A. Zhao, and S. Follmer, "shapeShift: A Mobile Tabletop Shape Display for Tangible and Haptic Interaction," in Adjunct Publication of the 30th Annual ACM Symposium on User Interface Software and Technology, 2017, pp. 77-79.

[21] E. Vonach, C. Gatterer, and H. Kaufmann, “VRRobot: Robot actuated props in an infinite virtual environment," in Proc of the IEEE Conference on Virtual Reality, 2017, pp. 74-83. 


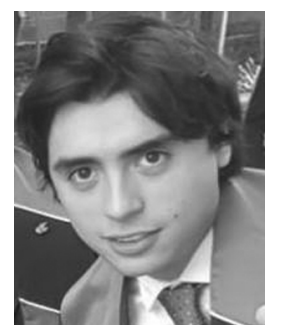

Víctor Mercado received the MS in intelligent systems from the Monterrey Institute of Technology and Higher Education, in Monterrey, Mexico in 2018. He is a doctoral student working in the Hybrid research team in Inria Rennes, France. His main research interests include encountered type haptic displays, human-computer interaction, and virtual reality.

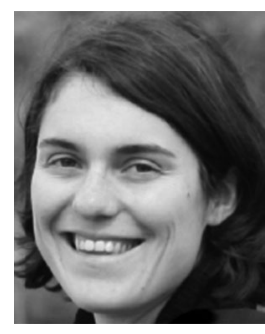

Maud Marchal received the MS and PhD degrees in computer science from the University Joseph Fourier, in Grenoble, France, in 2003 and 2006, respectively. She is an associate professor with the Computer Science Department, INSA (Engineering School) in Rennes, France. Her main research interests include physically based simulation, haptic rendering, 3D interaction, and virtual reality.

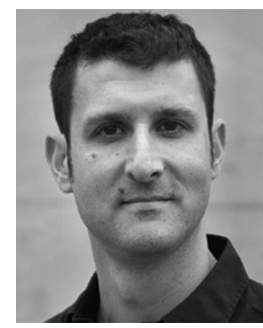

(2012-2013).
Anatole Lécuyer is a senior researcher and head of Hybrid research team, Inria Rennes, France. He is currently an associate editor of the IEEE Transactions on Visualization and Computer Graphics, the Frontiers in Virtual Environments and the Presence journals, and formerly of the ACM Transactions on Applied Perception and the International Journal of Humanities and Cultural Studies. He was a program chair of the IEEE Virtual Reality Conference (2015-2016) and the IEEE Symposium on 3D User Interfaces 